\title{
Neurocysticercosis (NCC) in 15-Year-Old Girl, East Nusa Tenggara, Indonesia: A Case Report
}

\author{
Albert William Hotomo*, Defranky Theodorus, Ivan Veriswan \\ Department of Child Health, Umbu Rara Meha Hospital, Waingapu, Indonesia \\ Email address: \\ albertwilliamhotomo@gmail.com (A. W. Hotomo),dokter_franky@yahoo.com (D. Theodorus), ivaneris@gmail.com (I. Veriswan) \\ ${ }^{*}$ Corresponding author
}

\section{To cite this article:}

Albert William Hotomo, Defranky Theodorus, Ivan Veriswan. Neurocysticercosis (NCC) in 15-Year-Old Girl, East Nusa Tenggara, Indonesia: A Case Report. American Journal of Pediatrics. Vol. 7, No. 1, 2021, pp. 39-43. doi: 10.11648/j.ajp.20210701.19

Received: March 5, 2021; Accepted: March 23, 2021; Published: March 30, 2021

\begin{abstract}
Neurocysticercosis (NCC) is an acquired parasitic infection of the central nervous system (CNS) in humans caused by encysted pork tapeworm larvae, namely Taenia solium. NCC has been discovered to be the most common etiology of focal seizure among the pediatric population. The severe CNS sequelae by NCC might cause to poor quality of life, eventually lead to sudden death. In this study, we report a case of NCC in a fifteen-year-old girl. Case illustration: a fifteen-year-old girl suddenly experienced a focal seizure of the right hand, along with a decreased of consciousness. The duration of the seizure was less than five minutes. It recurred for three times since ten hours before admission to hospital. She admitted that it was the first unprovoked seizure in her life. There was no record of fever and cough. She had a history of eating pork in the rural region of Sumba Island. Physical examination demonstrated GCS E3V4M5, normal findings of general and neurological status. Computed Tomography (CT) scan with contrast revealed a hypodense calcified lesion appended by a cyst with a dot sign, located at the grey-white matter junction at left parietal region. These findings supported colloidal vesicular stage of NCC. The diagnosis of this patient was neurocysticercosis. Therefore, she was given albendazole $400 \mathrm{mg}$ bid and valproic acid $(15 \mathrm{mg} / \mathrm{kg} / \mathrm{day})$ for a month, and intravenous dexamethasone $(1 \mathrm{mg} / \mathrm{kg}$ ) with a maximum dose of $12 \mathrm{mg}$ qid. After a month of treatment, she did not either have any seizure or other complaints. CT scan evaluation demonstrated a radiological improvement. Summary: NCC is identified as one of the commonest causes of epilepsy and seizure. The combination between antiparasitic with another supportive therapy gives comprehensive treatment of NCC.
\end{abstract}

Keywords: Neurocysticercosis, Epilepsy, Computed Tomography

\section{Introduction}

Neurocysticercosis is an acquired parasitic infection of the central nervous system (CNS) in humans caused by encysted larvae of Taenia solium. NCC has been known as a neglected and preventable cause of serious neurological symptoms in developing countries. $[1,2]$ It shows an essential etiology of seizure among children in endemic countries. [3] The identification of NCC as the main cause of death is considerably high, included as a neurological diseases, such as cerebral oedema, hydrocephalus, and intracranial hypertension. [2]

The clinical manifestation of NCC is varied greatly, which is influenced by several factors; the location and amount of lesions in CNS, parasites biological status, and host inflammatory status. [4] Hence, the disease severity could be from an asymptomatic to symptomatic stage, such as epilepsy, arachnoiditis, cognitive disturbance, visual loss, hydrocephalus, migraine, behavioral alteration, stroke and increased intracranial pressure. [1, 3, 5-10] Epilepsy has been known to be the most prevalent manifestation of NCC. $[3,5$, $10]$

On the clinical grounds, it is a great challenge for the clinician for diagnosing NCC, as it does not have any classic clinical manifestation. [4, 11-13] Neuroimaging, such as CT and Magnetic Resonance Imaging (MRI), has been known to be a fundamental investigations in order to make the diagnosis of NCC. [5, 14]

As the clinical manifestations proven to be atypical, it might be very challenging for medical doctor in order to identify this disease. However there is some evidence which is very helpful for the clinicians to manage the patients who 
suffer this kind of disease. This study presents a case report of NCC in girl age 15 years old with highlight of diagnosing and treating problems which discovered in this patient.

\section{Case Illustration}

A 15-year-old girl admitted to the emergency department at Umbu Rara Meha Hospital, Waingapu with a focal seizure of the right hand since ten hours before admission. The patient was referred from Waibakul Hospital, Waibakul with a diagnosis of suspected epilepsy with the differential diagnosis of space occupying lesion (SOL) at the brain. The seizure was the first unprovoked seizure she experienced in her life, with clonic movement at the right hand, along with a decreased of consciousness. The duration of the seizure was less than five minutes. It recurred for three times since ten hours before admission to hospital. There was no report of previous seizures before. When seizure occurred, the patient did not suffer any fever and the history of fever before the seizure also denied.

The history of cough, contact with tuberculosis (TB) patient nearby her neighborhood, report of family illness, routine medication report, and remarkable history during pregnancy was denied. History of growth and development fit to age. Her immunization report was complete according to the national health ministry standard. She had a record of eating pork in the rural region of Sumba Island. Poor hygiene and sanitation have been reported among the rural region in Sumba Island.

Physical examination presented GCS E3V4M5, with a normal findings of general and neurological status. The laboratory result showed a mild eosinophilia with a value 733 cells $/ \mathrm{mm}^{3}$. CT-scan with contrast revealed hypodense calcified lesion appended by a cyst with a dot sign, located at the grey-white matter junction at left parietal region which supports colloidal vesicular stage of NCC (Figure 1a and 1b). Patient diagnosed with neurocysticercosis. The treatment of this patient was the combination of anti-parasitic, anti-convulsant, and anti-inflammation drugs. Albendazole, as the anti-parasitic drug, with a dosage $400 \mathrm{mg} / 12$ hours was given to this patient orally for a month. For the anti-convulsant, the patient was given valproic acid with a dosage $15 \mathrm{mg} / \mathrm{kg} /$ day orally for a month. Dexamethasone, as the anti-inflammation was given intravenously with a dosage $1 \mathrm{mg} / \mathrm{kg}$ and maximum dose of $12 \mathrm{mg} / 6$ hours for a day. After a month of therapy, she neither had any seizure or other complaints. CT scan evaluation revealed that there was still a hypodense lesion at the left parietal region without any calcification in that area. In comparison with the CT-scan before the treatment, it showed a radiological improvement (Figure 2a and b). Therefore, albendazole $400 \mathrm{mg} / 24$ hours orally was given for one more week and valproic acid 15 $\mathrm{mg} / \mathrm{kg}$ /day was given for two years as the anti-epileptic drug for this patient. The CT scan evaluation will be administered to the patient after one year of therapy. The patient and her family were educated to prepare any type of meat properly until it is perfectly cooked in order to avoid the parasitic infection, especially cysticercosis, and the parents were informed to bring their daughter monthly or when seizure happened to the Waibakul Hospital so the pediatrician could make a follow up of her health condition and to evaluate her anti-epileptic drug regimen.

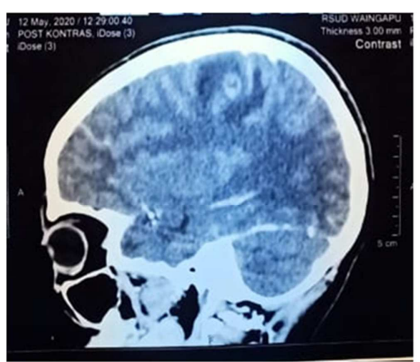

(a)

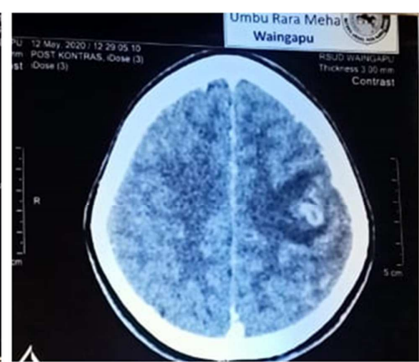

(b)
Figure 1. (a) and (b). a hypodense calcified lesion appended by a cyst with a dot sign, located at the grey-white matter junction at left parietal region from head CT-scan before the treatment was administered.

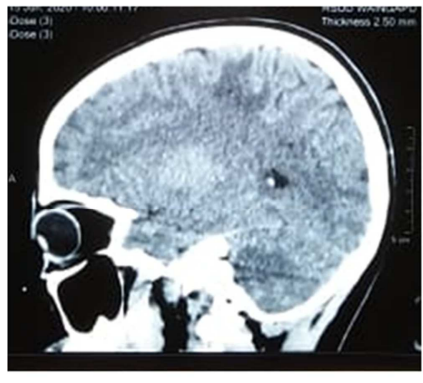

(a)

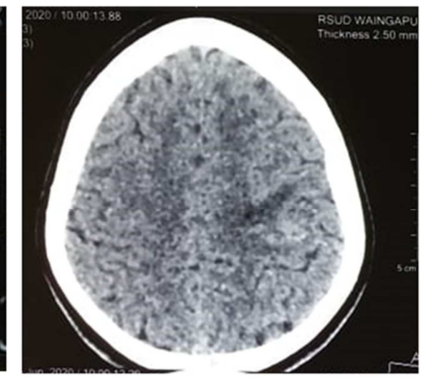

(b)
Figure 2. (a) and (b). a hypodense lesion without calcification at left parietal region from head CT-scan after one month administration of treatment.

\section{Discussion}

Neurocysticercosis has been identified as the commonest parasitic infection of the central nervous system. $[15,16]$ This case report discovered the most prevalent clinical manifestation of NCC, namely seizure. This patient suffered a sudden recurrent clonic focal seizure of the right hand which lasted for less than five minutes and accompanied with the decreased of consciousness. Studies have declared that seizure as the most frequent clinical manifestation of NCC. [3, 5, 10, 16] In addition, there were researches which discovered that focal seizure without generalization as the most common type of the seizure which caused by NCC. [17, 21] Furthermore, some researches have also proven that NCC as the etiology of late-onset epilepsy among pediatrics population, [18, 19] especially at the endemic regions which has been known that the parasitic infection was still high, like Southeast Asia. [3, 21]

The classic life cycle of T.solium consist of two types of host, human, as a definitive host and swine as an intermediate host. Individuals who consume undercooked pork meat, containing cysticerci will allow larvae to grow into adult tapeworm in the human's intestine (taeniasis). The adult tapeworm produces daily eggs which would be spread to the environment via feces. Therefore, in that condition, human acts as the definitive host. If the place have a poor hygiene and sanitation, then there might be a chance for roaming 
swine to consume food which is infected by eggs, then the life cycle in the intermediate host will begin. Eggs will develop into oncosphere (hexacanth embryos) by the impact of gastric and pancreatic enzyme. By using their hooklets, oncosphere will cross the intestinal wall, enter the bloodstream, and be delivered into various internal organs, such as brain, muscle, eye, subcutaneous tissue, etc. In the case of NCC, there is a disruption in the classic life cycle of T.solium which caused by individuals who inadvertently consume food or water contaminated by eggs. The parasite identifies human as an intermediate host and they will be carried in the systemic circulation which lead the migration of the parasite into the various internal organs, including the central nervous system. [3, 8, 20, 21] In this patient, we discovered that she had a history of eating pork in the rural area of Central Sumba. It is known that the method most people used to eat pork is not fully cooked, along with poor sanitation and hygiene. Hence, it might lead to the possibilities of $T$. solium infection in this patient.

The pleomorphic clinical manifestation of NCC leads to inconceivability for diagnosing NCC suspected cases on clinical findings merely. [2, 4, 21] Neuroimaging modalities, both CT scan and magnetic resonance imaging (MRI), are fundamental diagnostic tools in order to expose more specific characteristic of NCC features. It allows the visualization of T. solium development stage, namely vesicular, colloidal, granular-nodular, and calcified stage inside CNS, along with its localization and amount of lesions. [13, 15, 21] In comparison to MRI, CT is preferable beneficial to diagnose brain calcifications which is one of the most common finding of NCC, that might be hard to be visualized by using MRI. [22] At the vesicular stage, CT scan may visualize circumscribed, circular, hypodense areas, varying in size and quantity, with none contrast media enhancement. Whereas, MRI visualizes the vesicular larva presents with a CSF-like intensity signal on all sequences, without any encircling high signal on T2-weighted images. However, both CT and MRI might present a hyperdense about $2 \mathrm{~mm}$ mural nodule expressing the scolex of the parasite inside the vesicular cyst. As the cyst regression occurs, by using the CT scan with contrast media, it allows clinician to visualize an annular (colloidal phase) or nodular (nodular phase) enhancement lesion surrounded by irregular perilesional edema. While the cyst perishes, it might either vanish or become a dormant calcified nodule on CT. [15] Nevertheless, MRI has a higher sensitivity than CT for discovering the scolex and for diagnosing extraparenchymal NCC, particularly subarachnoid, ocular, and ventricular cysticercosis. [2, 15, 23] Serologic test appears to be inconclusive in NCC as several studies have discovered that subjects with inactive cysticercosis, especially brain calcifications or granulomas were also showed a negative result. $[4,12,15,24]$ In this patient, a hypodense calcified lesion appended by a cyst with a dot sign, located at the grey-white matter junction at left parietal region was discovered by using CT scan with contrast. These findings support colloidal vesicular stage of NCC. In this patient, MRI and serologic testing were not performed as they were not available yet at Sumba Island where the patient was treated.

Currently, significant breakthrough has been discovered in order to treat NCC. Albendazole has been proven to be the first drug of choice in treatment of viable intraparenchymal brain cyst. [4, 15, 20, 26] Its effectiveness to pass through the blood brain barrier has been confirmed, so that it has the capability for dealing with all locations of cysticerci. [3, 4, 20] The dosage of albendazole is $15 \mathrm{mg} / \mathrm{kg} /$ day in 2 divided doses, consumed after meal, and used for the duration of a month. However, several studies have revealed that the shorter duration of 8-14 days of albendazole consumption were found to be effective equivalently, particularly in intraparenchymal NCC. [3, 4, 25, 26] Albendazole is less expensive, safer with less side effects, better penetration to subarachnoid space. [20, 21] Even though praziquantel has been found to be a good substitute to albendazole for treating intraparenchymal cyst, it is much less potent in order to treat subarachnoid and ventricular cysticercosis as it showed an inadequate content of drug in these locations. [3] Furthermore, according to the clinical practice guidelines by the Infectious Diseases Society of America (IDSA) and the American Society of Tropical Medicine and Hygiene (ASTMH) 2017, it stated that albendazole, as a single antiparasitic treatment, for 10-14 days was recomended for patients who suffer 1-2 viable parenchymal cysticerci. The combination therapy between albendazole $(15 \mathrm{mg} / \mathrm{kg} / \mathrm{day})$ and praziquantel $(50 \mathrm{mg} / \mathrm{kg} /$ day $)$ for $10-14$ days was recommended particularly for patients with more than 2 viable parenchymal cysticerci. [27] In this case report, as the patient suffered one viable intraparenchymal NCC, so albendazole (with a dosage of $15 \mathrm{mg} / \mathrm{kg} / \mathrm{day}$ ) as a monotherapy was given in two divided dose with meal in the period of one month.

Corticosteroids have been proven to be very effective for overcoming local edema and inflammation which might be induced by the destruction of the parasites by the application of antiparasitic drugs. [3, 4, 5, 25] Additionally, steroid has the capability to increase the plasma content of albendazole, which are not appeared at praziquantel groups. [4, 14, 20, 21] Moreover, based on clinical practice guidelines by IDSA and ASTMH 2017, it has been affirmed that steroid as the adjunctive therapy which is administered prior to antiparasitic drugs. The current evidences which stated the dosage of corticosteroid are still unclear. One study comparing intravenous dexamethasone $6 \mathrm{mg}$ /day for 10 days with $8 \mathrm{mg} /$ day for 28 days followed by a taper, noted a higher seizure-free in the higher-dose group. [27] Other researches using oral prednisolone $2 \mathrm{mg} / \mathrm{kg} /$ day after meal which were started 2-3 days prior to albendazole, and continue it for the next 3-4 days.[21] In addition, other studies used steroids in concomitant with albendazole as a single daily dose of either oral prednisone (50 to $100 \mathrm{mg}$ ), or dexamethasone $(10 \mathrm{mg}$ ) intramuscularly. [1, 4] In this patient, intravenous dexamethasone was administered with a dosage $1 \mathrm{mg} / \mathrm{kg} /$ day and maximum dose of $12 \mathrm{mg} / 6$ hours for a day which was given inhospital. 
In general, most of the patients who suffer seizure secondary to NCC respond well with the monotherapy of first-line antiepileptic drug (AED), such as carbamazepine, valproate, or phenytoin. [27, 28, 29] In addition, several studies have revealed that by initiating the first-line AED gave a sufficient seizure control. [5, 21, 25] One study has discovered that carbamazepine and phenytoin were generally used in endemic countries due to their cost and availability. [28] Garcia et al have explained that NCC patients who suffer seizure have to get AED therapy for the duration of 2 years after the last seizure, and followed by continuous tapering off. [25] However, this patient will be administered with valproic acid ( $15 \mathrm{mg} / \mathrm{kg} /$ day) orally for two years after the last seizure as if the treatment of epilepsy, with a gradually monitoring of the side effects. This AED treatment regimen is in accordance with the clinical practice guidelines by IDSA and ASMTH 2017 which recommended about the management using AED therapy in all NCC patients with seizures and tapered it off after there is absence of seizure for 24 consecutive months along with the resolution of cystic lesion on imaging studies. Moreover, as there is lack of controlled data, the option for AED should be based on the local availability, cost, drug interactions, and potential side effects. [27]

To summarize treatment plan for this patient, that the cystic lesion evaluation by using the $\mathrm{CT}$ scan will be done after one year subsequently the initial seizure. EEG is not performed in this patient as it is not available yet at Sumba Island where the patient is treated now. Education about sanitation and hygiene is one of the most noteworthy prevention plan for this patient and for the community, as NCC is a highly preventable disease.

\section{Summary}

Neurocysticercosis is a CNS infection caused by T. solium tapeworm cyst and current evidences have revealed that NCC is one of the most frequent causes of seizure among children and adolescence. Poor hygiene and sanitation are the important epidemiological factors which trigger the course of this disease. Although NCC has a wide clinical spectrum, seizure has been discovered to be the most common clinical manifestation. Hence, neuroimaging is very essential tools in order to diagnose NCC. The management of NCC consists of antiparasitic drug in combination with other supportive therapy. By increasing the concern about hygiene and sanitation, along with the education to prepare pork well, we believe NCC is highly preventable. The collaboration between public health and other multidisciplinary sector is the key to eradicate this disease.

\section{References}

[1] Fogang YF, Camara M, Diop AG, Ndiaye MM. Cerebral neurocysticercosis mimicking or comorbid with episodic migraine?. BMC Neurology. 2014; 14: 138.
[2] Martins-Melo FR, Ramos Jr. AN, Cavalcanti MG, Alencar CH, Heukelbach J. Neurocysticercosis-related mortality in Brazil, 2000-2011: Epidemiology of a neglected neurologic cause of death. Acta Tropica. 2016: 128-136.

[3] Raffaldi I, Scolfaro C, Mignore F, Aguzzi S, Denegri F, Tovo PA. An uncommon cause of seizures in children living in developed countries: neurocysticercosis -a case report. Italian Journal of Pediatrics. 2011; 37: 9.

[4] Sotelo J. Clinical manifestations, diagnosis, and treatment of neurocysticercosis. Current Neurology and Neuroscience Reports. 2011; 11: 529-535.

[5] Takayanagui O. Neurocysticercosis. Arquivos de neuro-psiquiatria. 2013; 71 (9B): 710-713

[6] Kim S, Wang HS, Ju CI, Kim D. Acute hydrocephalus caused by intraspinal neurocysticercosis: case report. BMC Research Notes. 2014; 7: 2.

[7] Fugate JE, Lyons JL, Thakur KT, Smith BR, Hedley-Whyte ET, Mateen FJ. Infectious causes of stroke. Lancet Infectious Disease. 2014; 14: 869-880.

[8] Musara A, Soko N, Shamu S. Suprasellar cysticercosis cyst with optic nerve compression masquerading as an arachnoid cyst. Middle East African Journal of Ophtalmology. 2019; 114-116.

[9] Jain RS, Nagpal K, Handa R. Reversible dementia as presenting manifestation of racemose neurocysticercosis. Annals of Indian Academy of Neurology. 2013; 16 (3): 452-453.

[10] Moyano LM, Saito M, Montano SM, Gonzalvez G, Olaya S, Ayvar V, et al. Neurocysticercosis as a cause of epilepsy and seizures in two community-based studies in a cysticercosis-endemic region in Peru. PLOS Neglected Tropical Diseases. 2014; 8 (2): e2692.

[11] White, AC. Neurocysticercosis: updates on epidemiology pathogenesis, diagnosis, and management. Annual Review of Medicine. 2000: 187-206.

[12] Sinha S, Sharma BS. Neurocysticercosis: a review of current status and management. Journal of Clinical Neuroscience. 2009: 16 (7); 867-876.

[13] Moskowitz J, Mendelsohn G. Neurocysticercosis. Archives of Pathology and Laboratory Medicine. 2010; 134 (10); 1560-3.

[14] Coyle CM, Tanowitz HB. Diagnosis and treatment of neurocysticercosis. Interdisciplinary perspectives on infectious diseases. 2009: 180742.

[15] Carpio A, Romo ML, Parkhouse RME, Short B, Dua T. Parasitic diseases of the central nervous system: lessons for clinicians and policy makers. Expert Review of Neurotherapeutic. 2016; 16 (4): 401-414.

[16] Carpio A. Neurocysticercosis: an update. The Lancet Infectious Diseases. 2002; 2 (12): 751-762.

[17] Herrick JA, Maharathi B, Kim JS, Abundis GG, Garg A, Gonzales I, et al. Inflammation is a key risk factor for persistent seizures in neurocysticercosis. Annals of Clinical and Translational Neurology. 2018; 5 (5): 630-639.

[18] Wagner RG, Newton CR. Do helminthes cause epilepsy? Parasite Immunol. 2009; 31: 697-705. 
[19] Quet F, Guerchet M, Pion SD, Ngoungou EB, Nicoletti A, Preux PM. Meta-analysis of the association between cysticercosis and epilepsy in Africa. Epilepsia. 2009; 51: 830-837.

[20] Malla BA, Maqbool I, Lone SA, et al. An overview on neurocysticercosis. Journal of Pharmacology and Phytochemistry. 2018; 7 (6): 303-312.

[21] Singhi P, Saini AG. Pediatric neurocysticercosis: current challenges and future prospects. Pediatric Health, Medicine and Therapeutics; Macclesfield. 2016; 7: 5-16.

[22] Carpio A, Fleury A, Hauser WA. Neurocysticercosis: Five new things. Neurology Clinical Practice. 2013; 3 (2): 118-125

[23] Abdel Razek AA, Watcharakorn A, Castillo M. Parasitic diseases of the central nervous system. Neuroimaging Clinics of North America. 2011 Nov; 21 (4): 815-41, viii.

[24] Alexander AM, Prabhakaran V, Rajshekhar V, et al. Long-term clinical evaluation of asymptomatic subjects positive for circulating Taenia solium antigens. Transactions of the Royal Society of Tropical Medicine and Hygiene. 2010; 104: 809-10.
[25] Garcia HH, Nash TE, Del Brutto OH. Clinical symptoms, diagnosis, and treatment of neurocysticercosis. The Lancet Neurology. 2014 Dec; 13 (12): 1202-15.

[26] Singh G, White Jr AC. Determining better treatments for neurocysticercosis. The Lancet Infectious Diseases. 2014 July; 14 (3): 658-9.

[27] White Jr AC, Coyle CM, Rajshekar V, Singh G, Hauser AW, Mohanty A, Garcia HH, Nash TE. Diagnosis and treatment of neurocysticercosis: 2017 clinical practice guidelines by the Infectious Diseases Society of America (IDSA) and the American Society of Tropical Medicine and Hygiene (ASTMH). Clinical Infectious Diseases. 2018; 66 (8): e49-e75.

[28] Nash TE, Garcia HH. Diagnosis and treatment of neurocysticercosis. Nature Reviews Neurology. 2011; 7: 584594.

[29] Garcia HH, Gonzalez AE, Gilman RH. Cysticercosis of the central nervous system: how should it be managed? Current Opinion of Infectious Diseases. 2011; 24: 423-427. 